\title{
Diploid-dependent regulation of gene expression: a genetic cause of abnormal development in fish haploid embryos
}

\author{
C Luo and B Li \\ Institute of Biology, Hunan Normal University, Changsha, Hunan 410006, People's Republic of China
}

\begin{abstract}
A diploid-dependent regulatory mechanism of gene expression for spatial patterning of the eye in vertebrates has been determined by analyzing the phenotypes of haploid goldfish embryos. There are two gene loci in charge of eye spatial patterning during embryonic morphogenesis. The expressional probability for each copy of the two genes in a set of chromosomes is $50 \%$. A pair of genes in two sets of
\end{abstract}

homologous or heterologous chromosomes is $100 \%$ and essential for normal gene expression. The haploid condition itself would result in the obstruction of gene expression and abnormal development because the diploid-dependent regulatory apparatus will regulate gene expression in a haploid embryo according to the same rule as in the diploid embryo. Heredity (2003) 90, 405-409. doi:10.1038/sj.hdy.6800263

Keywords: spatial patterning; gene expression; regulation; haploid; diploid; eye; fish

\section{Introduction}

The animal developmental program proceeds in a given precise pattern of space and time. This spatial and temporal pattern is controlled by a strikingly complex genomic regulatory system. Investigating the genomic regulatory system and mechanism are of fundamental importance for understanding both development and evolutionary bioscience.

Considerable investigation of the molecular basis of development has concentrated on individual genes' promoters and their various regulatory elements. However, the genomic regulatory system and general principles at the whole genome level have remained frustratingly elusive in the vertebrate. This is largely because of its extreme complication, the absence of an appropriate sample, and the method of experimental analysis.

According to genetic theory, the genes in one set of homologous chromosomes should be sufficient to encode all the structures and physiological functions of an individual. However, all of the artificially induced haploid embryos of amphibians (Hertwig, 1911; Fankhauser, 1945) and fish (Oppermann, 1913; Purdom, 1969; Nagy et al, 1978; Chourrout, 1980; Streisinger et al, 1981; Refstie et al, 1982; Taniguchi et al, 1986; Komen et al, 1991; Luo and Liu, 1991; Varadi et al, 1999; Galbusera et al, 2000) have shown abnormalities and have died during embryonic development. The abnormality and mortality of haploids has generally been assumed to be because of the presence of deleterious recessive lethal genes in the haploid genome (Fankhauser, 1945; Purdom, 1969;

Correspondence: C Luo, Institute of Biology, Hunan Normal University, Changsha, Hunan 410006, People's Republic of China.

E-mail: cluo@hunnu.edu.cn

Received 18 September 2002; accepted 29 January 2003
Thorggard, 1983). Although such recessive lethal alleles may contribute to the death rate, they do not appear to be the underlying problem. In special conditions, diploid mitotic gynogenes from eggs obtained from the same single spawning survive well and do not show the same untimely death of the haploids (Chourrout, 1982; Mair et al, 1987). This phenomenon suggests that the haploid syndromes and high mortality must be due more to the lack of homologous genes in pairs rather than being due to the presence of lethal alleles (Varadaraj, 1993). In other words, the haploid itself might result in the obstruction of gene expression in embryonic development in the vertebrate. Obviously, analyzing the phenotypes of haploid and diploid embryos, which share the same genetic background but differ in the number of sets of chromosomes, would provide a new method for investigating regulation of gene expression at the whole genome level.

Of all the organs of the vertebrate body - apart from the brain - the eye is the most complex, particularly in terms of its cell diversity, component tissues, and parts, which are united to form an optic instrument of amazing proficiency and efficiency. Also, the eye of the vertebrate is the organ that has been studied in greatest depth. The complex course of development has been fairly well worked out using classical embryological methods (Ston, 1960; Lopashov and Stroeva, 1961; Jacbson, 1966; Bloemendal, 1977; Turner and Cepko, 1987; Saha et al, 1989). All of the differentiated cells and parts of the eye arranged in a given spatial pattern can be identified easily. Recently, more than 29 transcription factors required for the development of the vertebrate eye have been identified (Jean et al, 1998; Haider et al, 2000; Horsford et al, 2001; Chow et al, 2001) and several mutations affecting development of the retina in zebrafish (Malicki et al, 1996; Malicki and Driever, 1999; Jensen et al, 2001; Vihtelic and Hyde, 2002) have been detected. 
Therefore, the morphogenesis of the eye is suitable for an experimental analysis to study the genetic causes of abnormality in haploid embryos of vertebrates.

\section{Materials and methods}

\section{Animals}

A white, a red, and a red cup inbred strain of goldfish, Carassius auratus, obtained from a goldfish-breeding farm, were employed in this study. In each experiment, all the eggs used both in tests and in controls were obtained from a single spawning. Common carp, Cyprinus carpio, were obtained from a local fish farm

\section{Production and identification of gynogenetic haploid embryos}

Haploid gynogenes of the goldfish were induced using a UV-irradiated sperm of common carp (Luo and Liu, 1991). The goldfish has a specific double tail encoded by a recessive gene, while the common carp has a common single tail encoded by a dominant allele. The tails of all the hybrid embryos of the goldfish and common carp are all single tails just like the tail of the parent common carp (Luo, unpublished observations). With this character, the gynogenetic haploid embryos could be identified easily and unequivocally from the normal and abnormal hybrid embryos at an early developmental stage.

In inbreeding and intergeneric hybrid diploid control groups, the eggs coming from the same spawning were fertilized with normal sperm coming from the same inbred goldfish line or common carp, respectively.

\section{Production and identification of mitogynogenetic diploid embryos}

The optimum parameters for chromosome set duplication in goldfish were determined by systematical cytological observation on the developmental procedure of the first cleavage with the method of histological section ( $\mathrm{Li}$ et al, unpublished result). After being stimulated to start development by UV-irradiated sperm, the activated eggs of goldfish were reared at $18^{\circ} \mathrm{C}$. Mitogynogenetic diploid embryos were produced by inhibiting the first mitosis of the activated haploid eggs around $40 \mathrm{~min}$ after activation with a heat shock of $40^{\circ} \mathrm{C}$ applied for $2 \mathrm{~min}$. The haploid embryos can be distinguished from the diploid gynogenetic embryos by their characteristic syndromes such as twisted edematous body and curved tail. The mitogynogenetic homozygous diploids could be identified easily and unequivocally from the normal and abnormal hybrid diploid embryos at an early developmental stage by their tails.

\section{Results}

Some gynogenetic haploid embryos of the goldfish could develop to the hatching stage. However, none of them survived beyond the feeding stage. No diploid embryo with double tail was observed in any of the tests in that the eggs were activated with UV-irradiated common carp sperm but not treated with heat shock. In general, almost all the organs showed abnormality in a gynogenetic haploid group, although some organs of a given individual of the gynogenetic haploid group appeared normal.

Abnormality of eyes of the haploid embryos could be identified easily by specific defects in the retinal pigmented epithelium (Figure 1a) at an early stage. Normal and abnormal eyes were all observed in gynogenetic haploid embryos. Both eyes of a given haploid individual were either normal or abnormal. No haploid individual with one normal eye and one abnormal eye was observed. Some differences among the abnormal eyes were observed. However, the differences of abnormalities could not be distinguished distinctly. Therefore, the abnormal eyes were not further
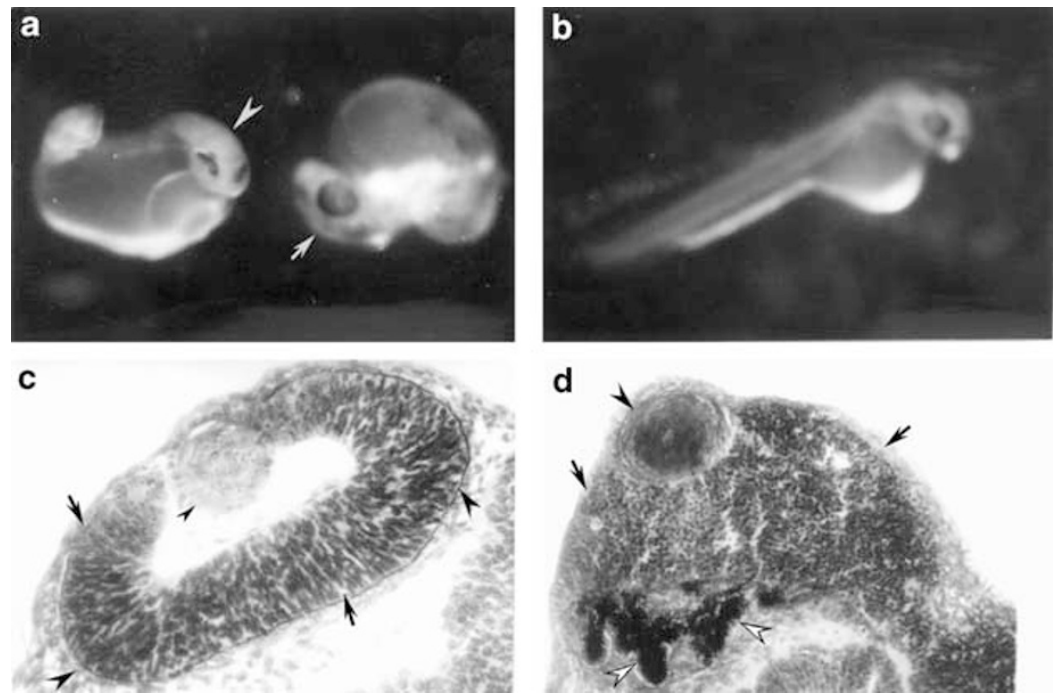

Figure 1 Normal and abnormal eyes of haploid goldfish embryos at hatching stage. (a) Haploid embryos with abnormal (arrowhead) or normal (arrow) eyes; for a given haploid embryo, the two eyes are either both normal or both abnormal. (b) A diploid embryo of control. (c) Microstructure of a normal eye of a haploid embryo, the differentiated cells, proportions, and the spatial pattern are just the same as that of the eye of the diploid embryo. Retinal pigmented epithelium: bigger arrowheads; neural retina: arrows; lens: smaller arrowhead. (d) Microstructure of an abnormal eye of a haploid embryo, the differentiated cells and proportions of the eye are not arranged in a correct spatial pattern. Retinal pigmented cells: white arrowheads; neural retina cells: black arrows; lens: black arrowhead. 
Table 1 Number of embryos with normal or abnormal eyes in gynogenetic haploid, mitogynogenetic diploid, inbred diploid, and intergeneric hybrid diploid

\begin{tabular}{|c|c|c|c|c|c|}
\hline Strains & Type of embryos & Total number & $\begin{array}{l}\text { Embryos with } \\
\text { abnormal eye }\end{array}$ & $\begin{array}{c}\text { Embryos with } \\
\text { normal eye }\end{array}$ & $\begin{array}{l}\text { Ratio of normal } \\
\text { and abnormal eyes }\end{array}$ \\
\hline \multirow[t]{4}{*}{ Red } & Gynogenetic haploid & 802 & 605 & 197 & $1: 3.02$ \\
\hline & Mitogynogenetic diploid & 60 & 0 & 60 & \\
\hline & Inbred diploid & 358 & 0 & 358 & \\
\hline & Intergeneric hybrid diploid & 336 & 0 & 336 & \\
\hline \multirow[t]{4}{*}{ White } & Gynogenetic haploid & 419 & 303 & 116 & $1: 2.60$ \\
\hline & Mitogynogenetic diploid & 48 & 0 & 48 & \\
\hline & Inbred diploid & 356 & 0 & 356 & \\
\hline & Intergeneric hybrid diploid & 425 & 0 & 425 & \\
\hline \multirow[t]{2}{*}{ Red cup } & Gynogenetic haploid & 3046 & 2255 & 791 & $1: 2.85$ \\
\hline & Inbred diploid & 318 & 0 & 318 & \\
\hline
\end{tabular}

All the haploid and diploid embryos were from eggs obtained from a single spawning in each test and were counted at the hatching stage. Haploid embryos were identified using both the characteristic syndromes of haploids and the double tail of goldfish. Abnormality of the eye was identified according to the characteristic defects of the retinal pigmented epithelium.

classified into subgroups and the variation was not further analyzed.

The results of a statistical analysis of the haploid individuals with normal or abnormal eyes shown in Table 1 are very surprising. In every test, about onefourth of the haploid gynogens developed normal eyes, the other three quarters developed obviously abnormal eyes. However, in the controls of mitogynogenetic diploids, inbred diploids, and intergeneric hybrid diploid embryos, no specific abnormal eyes, as seen in the haploid embryos, were observed in any of the tests.

On the histological sections of the embryonic eyes, the microscopic structure of the normally developed eyes of the haploid embryos was just like the structure of the control diploid embryos at the same stages (Figure 1c). The abnormal eyes of the haploid embryos had also developed all of the differentiated cellular types and parts of the vertebrate eye, such as pigment retina cells, neural retina cells, lens, and cornea. However, these differentiated cells or parts were not arranged in the correct positions (Figure 1d). No optical cup was formed on any of the observed abnormal eyes of the haploid gynogenes. The pigmented cells, which should be distributed evenly on the whole outer layer of the optical cup and form the retinal pigmented epithelium in the normal eye, assembled in a small region. The neural retina cells, which should constitute the inner layer of the optical cup, were in a disordered state. There was no cup between the lens and the neural retina cells in any of the abnormal eyes examined from haploid embryos. These results indicated that the abnormal development of the eye in haploid embryos did not result from abnormal differentiation but from an abnormal positional arrangement of the differentiated cells and parts.

\section{Discussion}

Using two different species of fish that have distinct genetic marks, the possibility of paternal contamination can be excluded. Meanwhile, all the gynogenetic haploid embryos had died at the first-feeding stage and no spontaneous gynogenetic diploid individual has been observed. These observations suggest that meiosis is normal in these fish, and the gynogenetic offspring could not be a mixture of mitogynogenetic homozygous and spontaneous meiogenic heterozygous individuals.

The observed ratio of about one to three has revealed that, during the development of the eye in the goldfish, there are two different genes in charge of the spatial positional arrangement of the differentiated cells and parts. Since there is only one set of chromosomes in haploid embryos, if there were two allelic genes, one dominant wild type and the other a deleterious recessive mutation, the ratio of haploid embryos with normal and abnormal eyes would be about one to one. Therefore, any two genes involved are not alleles of the same genetic locus but different alleles at two gene loci. Since no abnormal eyes appeared in either the controls of mitogynogenetic diploid embryos or by inbreeding diploid embryos that shared the same genetic background as the haploid embryos, it is impossible that there could have been deleterious recessive alleles of both gene loci in all of the three inbred strains. The normal and abnormal phenotypes of the eye therefore do not reflect genotype, but rather, the expressional state of the two genes. It appears that some obstruction of gene expression in the haploid has resulted in an abnormal spatial pattern of the eye.

Consequently, the ratio of about one to three also indicates that each copy of the two regulator genes in a set of chromosomes only has a $50 \%$ chance of being selected for expression, both in haploid and diploid embryos. In diploid embryos, the combined expression probability for a pair of genes is $100 \%$. Therefore, there would be only one composite type of gene expression that both genes express and all the eyes are normal. In haploid embryos, there would be two possible expression states for a gene. That is to say, that a gene could be either expressed or silent. Since the eye spatial patterning is arranged by two genes as reasoned above, there would be four possible composite expression types according to mathematical logic (Figure 2). Only the type in which both the two genes are expressed would develop normal eyes. The probability that the two genes would be simultaneously expressed is one-fourth of the haploid 

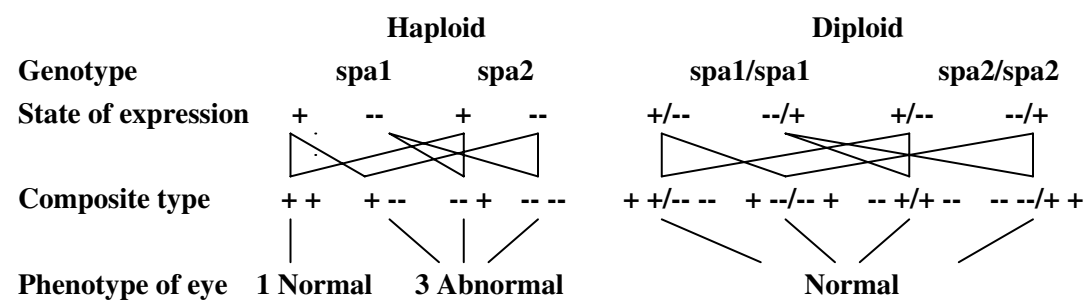

Figure 2 Expression of two spatial arranging genes (spa) and relative phenotypes of eyes in haploid and diploid embryos of goldfish. The two genes in charge of spatial pattern arrangement of the eye were named spa1 and spa2. There was $1 / 2$ probability for every copy of the two genes in a set of chromosomes to be selected for expression both in haploids and in diplods. In haploid conditions, single copy of a gene is either in expression $(+)$ or in silence $(-)$ condition by chance. Therefore, there would be four different composite types in terms of gene expression $(++,+-,-+,--)$ for the two genes in haploid embryos. Only one type in which the two genes, both were selected for expression $(++)$ would develop normal eyes. The other three-fourths would develop abnormally for either one $(+-,-+)$ or both $(--)$ of the two genes were not selected for expression. In diploid conditions, there was a 100\% probability for the pair of genes to be selected for expression $(+/-$ or $1-/+)$. Therefore, there would be only one composite type where both genes are selected for expression and all the diploid embryos develop normal eyes.

embryos. As a result, there would be only one-fourth of the haploid embryos that develop normal eyes. The other three types of haploid embryos develop abnormal eyes because at least one of their two regulator genes was not expressed.

The results suggest that, in embryonic morphogenesis of vertebrates, (1) there are some genes responsible for the spatial patterning, and (2) the regulatory mechanism of expression of these spatial-control genes is not based on one but two sets of chromosomes. Vertebrates have established an ingenious regulatory apparatus to modulate transcription of the genes on the two sets of chromosomes in a precisely logical manner during evolution. In haploid, the diploid-dependent regulatory apparatus selects a gene for expression according to the same rule as in diploid, which results in some genes not being selected for expression. This may be the cause why haploidy itself can result in obstruction of gene expression and developmental abnormality, at least of the spatial pattern formation of the eye in vertebrates.

Recently, Jensen et al (2001) have isolated two mutations of the mosaic eyes gene in which the retinal organization is severely disrupted. The mutations of the mosaic eyes gene cause retinal pigmented epithelium abnormalities, retinal abnormalities, reduced brain ventricles, circulation abnormalities, and dorsal curvature of the tail, but do not cause abnormal retinal cell differentiation. These phenotypes are similar to the phenotypes of those haploids. We reason that the mosaic eyes gene might be one of the spatial patterning control genes affected in the haploids.

The diploid-dependent regulatory apparatus and its nature have not yet been fully investigated. The reason why the regulatory apparatus modulates gene expression according to precise mathematical logic, but cannot distinguish the haploid and diploid conditions is very confusing and interesting.

One possible explanation might be based on imprinting of DNA methylation. In mammals, it has been demonstrated that the presence of methylated cytosines in the promoter of a gene correlates with the repression of transcription from that gene (van der Ploeg and Flavell, 1980; Groudine and Weintraub, 1981; Bussslinger et al, 1983). The pattern of methylation on a given gene can differ between egg and sperm (Barlow et al, 1991; DeChiara et al, 1991; Bartolomei and Tilghman, 1997).
This maternal imprinting may be responsible for the abnormal development of the gynogenetic goldfish haploids, since some genes, inherited from the mother, are switched off by methylation. In this case of observations, two unlinked gene loci being required in active form for eye development, one could imagine that, in the mother's meiosis, homologous pairs of the genes at both loci have one of their alleles switched off by methylation, and, the alleles then seggregate into the ovum. Therefore, a haploid embryo would only have a one-in-four chance of receiving the active alleles at both loci. However, such a model would raise questions as to why diploids always have at least one active gene copy. One hypothesis could be that the repression of the female's genome by methylation is removed at the moment of fertilization. However, in the case of all the eggs used in each experiment being obtained from a single spawning, if the methylation is erased at fertilization, the maternal imprinting introduced onto the genome during meiosis should be the same. Therefore, this hypothesis would raise a second question as to how the females could have one but only one inactivated copy of some genetic loci. We further hypothesize that only if all the imprinting of the maternal genome is removed at fertilization, the methylation information imprinted on the paternal genome remain so throughout development, and are passed on in their imprinted state by their daughters. The hypothesis would also raise a third question as to why the maternal-inherited methylation information is not erased in induced gynogenetic haploids. A third hypothesis is required: the activation effect with UVirradiated sperm could not have the effect of removing the repression of maternal-inherited alleles that is postulated to occur at fertilization.

The diploid-dependent regulatory apparatus and its nature might have something to do with the cisregulatory system. A very interesting promoter that operates in a logical manner has been reported (Arnone and Davidson, 1997; Yuh et al, 1998). Experimental and computational analysis revealed that the logical functions of the Endo16 cis-regulatory system are all dependent on explicitly specified DNA sequences in the genome. Perhaps the vertebrates also have evolved specified DNA in their genome for mediating the diploid-dependent regulatory apparatus to process in logical manner. 


\section{Acknowledgements}

We thank Hongbo Wen, Jifang Liu, Mingmin Huang, and Rurong Zhao for identifying and counting part of the haploid embryos. The work was supported by the National Science Foundation of China (NSFC-39830300) and the Ministry of Education, People's Republic of China.

\section{References}

Arnone MI, Davidson EH (1997). The hardwiring of development: organization and function of geneomic regulatory systems. Development 124: 1851-1864.

Barlow DP, Storger R, Harrmann BG, Saito K, Schweifer N (1991). The mouse insulin-like growth factor type-2 receptor is imprinted and closely linked to the Tme locus. Nature 349: 84-87.

Bartolomei MS, Tilghman SM (1997). Genomic imprinting in mammals. Annu Rev Genet 31: 493-525.

Bloemendal H (1977). The vertebrate eye lens - a useful system for the study of fundamental biology processes on a molecular level. Science 197: 127-138.

Busslinger M, Hurst J, Flavell RA (1983). DNA methylation and the regulation of globin gene expression. Cell 34: 197-206.

Chourrout D (1980). Thermal induction of diploid gynogenesis and triploid in the eggs of the rainbow trout. Nutr Dev 20: 727-733

Chourrout D (1982). Gynogenesis caused by ultraviolet irradiation of samonid sperm. J Exp Zool 223: 175-181.

Chow RL, Snow B, Novak J, Looser J, Freund C, Vidgen D et al (2001). Vsx1, a rapidly evolving paired-like homeobox gene expressed in cone bipolar cells. Mech Dev 109: 315-322.

DeChiara TM, Robertson EJ, Efstratiadis A (1991). Parental imprinting of the mouse insulin-like growth factor II gene. Cell 64: 849-859.

Fankhauser G (1945). The effects of changes in chromosome number on amphibian development. $Q$ Rev Biol 20: 20-78.

Galbusera P, Volckaert FAM, Ollevier F (2000). Gynogenesis in the African Catfish Clarias gariepinus (Burchell, 1822) III. Induction of endomitosis and the presence of residual genetic variation. Aquaculture 185: 25-42.

Groudine M, Weintraub H (1981). Activation of globin genes during chick development. Cell 24: 393-401.

Haider NB, Jacobson SG, Cideciyan AV, Swiderski R, Streb LM, Searby $C$ et al (2000). Mutation of a nuclear receptor gene, NR2E3, causes enhanced S cone syndrome, a disorder of retinal cell fate. Nat Genet 24: 127-131.

Hertwig O (1911). Die Radiumkrankheit tierischen Kiemrellen. J Areh Mikrosk Anat 77: 1-97.

Horsford DJ, Hanson I, Freund C, Mcinnes RR, Van Heyningen $\mathrm{V}$ (2001). Transcription factors in eye disease and ocular development. In: Scriver CR (ed) The Metabolic and Molecular Basis of Inherited Disease, McGraw-Hill: New York. vol. 4, pp 5987-6032.

Jacbson AG (1966). Inductive processes in embryonic development. Science 152: 25-34.

Jean D, Ewan K, Gruss P (1998). Molecular regulators involved in vertebrate eye development. Mech Dev 76: 3-18.

Jensen AM, Walker C, Westerfield M (2001). Mosaic eyes: a zebrafish gene requied in pigmented epithelium for apical localization of retinal cell division and lamination. Development 128: 95-105.
Komen J, Bongers ABJ, Richter CJJ, Van Muiswinskel WB, Huisman EA (1991). Gynogenesis in common carp (Cyprinus carpio): II. The production of homozygous gynogenetic clones and F1 hybrids. Aquaculture 92: 127-142.

Lopashov GV, Stroeva OG (1961). Morphogenesis of the vertebrate eye. Adv Morphog 1: 331-370.

Luo C, Liu Y (1991). Studies on production of gynogenetic grass carp and crucian carp. Act Sci Nat Univ Norm Hun 14(2): 154159.

Malicki J, Driever W (1999). Oko meduzy mutations affect neuronal patterning in the zebrafish retina and reveal cellcell interactions of the retinal neuroepithelial sheet. Development 126: 1235-1246.

Malicki J, Neuhauss SCF, Schier AF, Solnica-Krezel L, Stemple DL, Stainier DYR et al (1996). Mutations affecting development of zebrafish retina. Development 123: 263-273.

Mair GC, Scott AG, Beardmore JA, Skibinski DOF (1987). A technique for induction of diploid gynogenesis in Oreochromis niloticus by suppression of the first mitotic division. In: Tiews K (ed) Proceedings of World Symposium on Selection, Hybridization and Genetic Engineering in Aquaculture, Bardeaux: Berlin. pp 290-299.

Nagy A, Rajki K, Horvath L, Csanyi V (1978). Investigation on carp. Cyprinus carpio L. gynogenesis. J Fish Biol 13: 214-224.

Oppermann K (1913). Die Entwicklung von Forelieneiern nach Befruchtung mit radium bestrahlten Samemfaden. Arch Mikrosk Anat 83: 141-189.

Purdom CE (1969). Radiation-induced gynogenesis and androgenesis in fish. Heredity (Edinburgh) 24: 431-434.

Refstie T, Stoss J, Donoldson EM (1982). Production of all female Coho salmon (Oncorhynchus kisutch) by diploid gynogenesis using irradiated sperm and cold shock. Aquaculture 29: 67-82.

Saha M, Spann CL, Grainger RM (1989). Embryonic lens induction: more than meets the optic vesicle. Cell Differ Dev 28: $153-172$.

Ston LS (1960). Polarization of the retina and development of vision. J Exp Zool 145: 85-93.

Streisinger G, Walker C, Dower N, Knauber D, Singer F (1981). Production of clones of homozygous diploid zebra fish (Brachydanio rerio). Nature 291: 293-296.

Taniguchi N, Kijima A, Fukai J, Inada Y (1986). Conditions to induce triploid and gynogenetic diploid in Ayu (Plecoglossus altivilus). Bull Jpn Soc Sci Fish 52: 49-53.

Thorggard GH (1983). Chromosome set manipulation and sex control. In: Hoar WS, Randall DJ, Donadlson EM (eds) Fish Physiology, Academic Press: New York. Vol. 9B, pp 405-434.

Turner DL, Cepko CL (1987). A common progenitor for neurons and glia persists in rat retina late in development. Nature 328 131-136.

Varadaraj K (1993). Production of viable haploid Oreochromis mossanbicus gynogenes using UV-irradiated sperm. J Exp Zool 267: 460-497.

Van Der Ploeg LHT, Flavell RD (1980). DNA methylation in the human $\gamma-\delta-\beta$ globin locus in erythroid and non-erythroid cells. Cell 19: 947-958.

Varadi L, Benko I, Varga J, Horvath L (1999). Induction of diploid gynogenesis using interspecific sperm and production of tetraploids in African catfish, Clarias gariepinus Burchell (1822). Aquaculture 173: 401-411.

Vihtelic TS, Hyde DR (2002). Zebrafish mutagensis yields eye morphological mutants with retinal and defects. Vision Res 42: 535-540.

Yuh C-H, Boulouri H, Davidson EH (1998). Genomic cisregulatory logic: experimental and computational analysis of sea urchin gene. Science 279: 1896-1902. 